\title{
Reporte de consumo de plantas medicinales en gestantes del Centro de Salud Viña Alta, La Molina. Lima, Perú
}

Echevarría-Merino H ${ }^{1, a}$; Flores-Asenjo W 1,a ; Garay-Ortega J 1,a; Roca-Moscoso MA 1,2,b; Salazar-Granara A* 1,3,c

\section{RESUMEN}

El objetivo de este trabajo fue conocer la frecuencia y la manera en que las plantas medicinales son empleadas por las gestantes del Centro de Salud Viña Alta del distrito de La Molina. En el estudio participaron veintiuna gestantes, quienes respondieron una encuesta, validada por expertos, sobre el uso de plantas medicinales. Se demostró que el 86,00 \% de las gestantes utilizaron plantas medicinales, y que $67,00 \%$ no consultó con un médico sobre su empleo. La manera más frecuente de consumo de las plantas medicinales fue la vía oral, en forma de infusiones. Las plantas medicinales más utilizadas fueron manzanilla, perejil, eucalipto, boldo, aloe, menta y ruda; y algunas de ellas mostraron efecto teratogénico, estimulante uterino, abortivo, entre otros. Es necesario conocer la cantidad real y la manera más frecuente en que las gestantes consumen las plantas medicinales, con la finalidad de clasificar estos productos de acuerdo al nivel de seguridad que ofrecen y promover el uso racional de la medicina tradicional para contribuir en la salud pública.

Palabras clave: Plantas medicinales; Mujeres embarazadas; Teratogénesis; Abortivos (Fuente: DeCS BIREME).

\section{Report on the use of medicinal plants among pregnant women treated at the Centro de Salud Viña Alta, La Molina. Lima, Peru}

\section{ABSTRACT}

This research aimed to find out the frequency and ways of using medicinal plants by pregnant women treated at the Centro de Salud Viña Alta, located in La Molina district. Twenty-one (21) pregnant women voluntarily participated in the study, where they were administered an expert-validated survey regarding the use of medicinal plants. The results showed that $86 \%$ of the pregnant women used medicinal plants and $67 \%$ did not ask their doctor before using them. The most frequent way of using the medicinal plants was by oral intake as herbal teas. The most widely used medicinal plants were chamomile, parsley, eucalyptus, boldo, aloe, mint and rue, some of which produced a teratogenic effect, uterine stimulant action, abortifacient effect, among others. It is necessary to know the actual quantity and most frequent way of using medicinal plants by pregnant women to classify these products as for its safety level and promote the rational use of traditional medicine to contribute to public health.

Keywords: Plants, medicinal; Pregnant women; Teratogenesis; Abortifacient agents (Source: MeSH NLM).

1 Universidad de San Martín de Porres, Facultad de Medicina Humana, Centro de Investigación de Medicina Tradicional y Farmacología. La Molina, Lima, Perú.

2 Sociedad Científica de Estudiantes de Medicina de la Universidad de San Martín de Porres (SOCIEM-USMP). La Molina, Lima, Perú.

3 Sociedad Peruana de Farmacología y Terapéutica Experimental (SOPFARTEX). San Borja, Lima, Perú.

a Médico Cirujano.

b Estudiante de Medicina Humana.

c Doctor en Medicina.

* Autor corresponsal. 


\section{INTRODUCCIÓN}

El uso de medicamentos durante la gestación presenta una serie de limitaciones debido al escaso conocimiento de la farmacocinética y farmacodinamia en esta etapa de vida de la mujer, ya que el proceso de síntesis de un nuevo fármaco excluye, por ética, a las gestantes.

Otro aspecto a considerar es la exposición del nuevo ser a la acción del medicamento, lo que se denomina efecto teratógeno potencial. Este efecto se presenta cuando se expone al feto a un determinado producto, como un fármaco, en las etapas tempranas de la gestación, lo que puede provocar abortos, embriotoxicidad o fetotoxicidad. Estas últimas se manifiestan como malformaciones congénitas, autismo, cáncer, entre otros. Por ello, si un medicamento se emplea durante el embarazo, debe ser bajo la prescripción y control del médico tratante ${ }^{(1)}$.

Una investigación multicéntrica que incluye Europa, América del Norte, Australia, Asia y Sudamérica revela que las mujeres gestantes utilizan las plantas medicinales con frecuencia, en un rango de $4,3 \%$ hasta $69 \%$ (2). Como se sabe, estos productos tienen propiedades farmacológicas y son de fácil acceso para la población por poseer múltiples usos y diversas presentaciones ${ }^{(3)}$.

Las propiedades de una planta medicinal se deben a diversos metabolitos secundarios en su composición, lo cual es reconocido por la Organización Mundial de la Salud (OMS), institución que también promueve el uso racional de las terapias procedentes de la medicina tradicional, la generación de evidencia acerca de sus usos y la reglamentación de su empleo por la población ${ }^{(4,5)}$. Por otra parte, aunque en el Perú existe una ley que regula el comercio de las plantas medicinales ${ }^{(6)}$, en la realidad el acceso a ellas se da de manera formal e informal ${ }^{(7,8)}$, y están disponibles en supermercados, farmacias, mercados de barrio, ambulantes, etc. ${ }^{(9)}$.
En este contexto, se suman estudios que demostraron efectos teratogénicos e inducción de abortos de plantas como la alfalfa, el aloe, caléndula, chinchona, ginger, valeriana, ruda, boldo, entre otros ${ }^{(10-12)}$. Por esto, ya que la gestación es una etapa muy especial en la vida de una mujer, y que se podría incurrir en prácticas empíricas para el cuidado de su salud sin consultar al médico ${ }^{(13)}$, el presente estudio preliminar indagó acerca del uso de plantas medicinales en un grupo de gestantes residentes de La Molina en Lima, Perú.

\section{EL ESTUDIO}

Estudio de corte transversal acerca del uso y la forma de empleo de plantas medicinales por parte de las mujeres embarazadas atendidas en el Centro de Salud Viña Alta del distrito de La Molina, en la región Lima (Perú), entre junio y octubre del 2014. Se empleó una encuesta mixta, validada por expertos, con preguntas sobre la etapa de gestación, el uso y formas de empleo de las plantas medicinales. El muestreo fue no probabilístico e incluyó a 21 gestantes mayores de 18 años que participaron luego de firmar el consentimiento informado. El estudio fue aprobado por el Instituto de Investigación de la Facultad de Medicina Humana de la Universidad de San Martín de Porres.

En la tabla 1 se muestra que el $86,00 \%(n=18)$ de las gestantes consumieron plantas medicinales. Según el periodo de gestación, la distribución de empleo de este producto fue la siguiente: $5,00 \%$ en el primer trimestre $(n=1) 33,00 \%$ en el segundo $(n=7)$ y $62,00 \%$ en el tercero $(n=13)$. Asimismo, se reporta que el $78,00 \%$ de las gestantes no consultan a su médico para emplear las plantas medicinales $(n=14)$. Además, se observó que las fuentes de información para el uso de la planta medicinal fueron la familia en el $78,00 \%$ de los casos $(n=14)$; los médicos, $11,00 \%(n=2)$; la televisión, $11,00 \%(n=2)$; los vecinos, $11,00 \%(n=2)$; y la radio, $6,00 \%(n=1)$.

Tabla 1. Características de las gestantes del centro de salud Viña Alta, La Molina, Lima-Perú

\begin{tabular}{|lcc|}
\hline Ítem de estudio & $\begin{array}{c}\text { N. }{ }^{\circ} \text { de } \\
\text { menciones }\end{array}$ & $\begin{array}{c}\text { Porcentaje } \\
(\%)\end{array}$ \\
\hline Consumo de plantas medicinales & & \\
\hline Sí & 18 & 86,00 \\
\hline No & 3 & 14,00 \\
\hline Consulta al médico uso de plantas medicinales & & \\
\hline Sí & 4 & 22,00 \\
\hline No & 14 & 78,00 \\
\hline
\end{tabular}




\begin{tabular}{|lcc|}
\hline \multicolumn{1}{|c|}{ ítem de estudio } & $\begin{array}{c}\text { N. }{ }^{\circ} \text { de } \\
\text { menciones }\end{array}$ & $\begin{array}{c}\text { Porcentaje } \\
(\%)\end{array}$ \\
\hline Fuente de información de las plantas medicinales & & \\
\hline Familia & 14 & 78,00 \\
\hline Médico & 2 & 11,00 \\
\hline Televisión & 2 & 11,00 \\
\hline Vecinos & 2 & 11,00 \\
\hline Radio & 1 & 6,00 \\
\hline Periodo de gestación & & \\
\hline Primer trimestre & 1 & 5,00 \\
\hline Segundo trimestre & 7 & 33,00 \\
\hline Tercer trimestre & 13 & 62,00 \\
\hline
\end{tabular}

Fuente: encuesta del estudio.

Las plantas medicinales que se consumieron con mayor frecuencia durante la gestación fueron manzanilla, en el $32,00 \%$ de la población $(\mathrm{n}=16)$; perejil, $20,00 \%(\mathrm{n}=10)$; y eucalipto, $16,00 \%(n=8)$. Asimismo, las principales formas farmacéuticas empleadas fueron la infusión en el $52,00 \%$ de casos $(n=20)$; la cocción, $26,00 \%(n=10)$; y el ungüento, $13,00 \%(n=5)$ (Tabla 2$)$.

Tabla 2. Plantas medicinales y formas uso empleadas por un grupo de gestantes del centro de salud Viña Alta, La Molina, Lima-Perú

\begin{tabular}{|lcc|}
\hline Iitem de estudio & $\begin{array}{c}\text { N. }{ }^{\circ} \text { de } \\
\text { menciones }\end{array}$ & $\begin{array}{c}\text { Porcentaje } \\
(\%)\end{array}$ \\
\hline Plantas medicinales & & \\
\hline Manzanilla (Matricaria chamomilla) & 16 & 32,00 \\
\hline Perejil (Petroselinum crispum) & 10 & 20,00 \\
\hline Eucalipto (Eucalyptus globulus) & 8 & 16,00 \\
\hline Boldo (Peumus boldus) & 6 & 12,00 \\
\hline Sábila (Aloe vera) & 4 & 8,00 \\
\hline Menta (Mentha piperita) & 3 & 6,00 \\
\hline Ruda (Ruta graveolens) & 2 & 4,00 \\
\hline Formas de uso & & \\
\hline Infusión & 20 & 52,00 \\
\hline Cocción & 10 & 26,00 \\
\hline Ungüento & 5 & 13,00 \\
\hline Inhalación & 3 & 6,00 \\
\hline Extracto & 1 & 3,00 \\
\hline
\end{tabular}

Fuente: encuesta del estudio.

\section{DISCUSIÓN}

En este estudio la frecuencia de uso de plantas durante la gestación fue de $86,00 \%(\mathrm{n}=18)$. Un estudio multicéntrico que incluyó 9459 gestantes de países de Europa, Asia, Australia, Norteamérica y Sudamérica mostró un rango de frecuencia de $4,3 \%$ a $69 \%$. El 17,9 \% correspondió a la población de Sudamérica, y de este grupo, 18 participantes provenían de Perú; esta investigación fue hecha mediante encuestas vía la página web de la OTIS (del inglés, Organization of Teratology Information Specialists). Por otra parte, un estudio reporta una frecuencia de uso de $81 \%$ en las gestantes de Italia; $69,5 \%$, en Rusia; $67,5 \%$, en Nigeria; y 56,8 \%, en Brasil ${ }^{(2,14)}$. 
Respecto a la forma de uso y administración de las plantas medicinales en las gestantes, en este estudio, la principal vía e ingesta fue oral, como infusión y cocción. Estos resultados son similares a los obtenidos en trabajos de Colombia, Brasil y Uganda ${ }^{(15-17)}$. La ingesta por vía oral implica un potencial efecto sistémico de la planta medicinal, posibilitando la aparición de efectos teratogénicos $\mathrm{u}$ abortivos, además de interacciones farmacológicas no deseadas. ${ }^{(10-12,18)}$.

Un punto importante es el asesoramiento sobre el uso de la planta medicinal durante la gestación. Observamos que el médico no es la fuente consultada para este fin, sino la familia, hecho que se reporta en otras investigaciones $(2,17,19)$. En nuestra investigación, la manzanilla es la planta medicinal más utilizada por las participantes, al igual que en otros estudios ${ }^{(2,10,14,18)}$. Durante la gestación, el consumo moderado del té de manzanilla es seguro; sin embargo, la ingesta repetida de grandes cantidades podría estimular al útero y ocasionar el cierre prematuro del conducto arterioso en el feto. La sábila y la ruda pueden estimular al útero y ser abortivos. La menta provocaría un sangrado uterino (efecto emenagogo). El boldo ha mostrado ser un potencial teratógeno y abortivo en ratas preñadas, y comparte esta última acción con el eucalipto; mientras que no se han reportado estos efectos en el perejil $(11,12,20)$.

Las limitaciones de este trabajo radican en el tamaño de la muestra y el sesgo de información. Sin embargo, este estudio alerta sobre el uso de las plantas por las gestantes, tema que debe ampliarse en el futuro. Para ello, sugerimos indagar con mayor precisión en la clínica el desenlace de la exposición, la corroboración de las plantas utilizadas y la comprobación de efectos negativos en el laboratorio. Este trabajo permite continuar con una línea de investigación poco explorada, que podría contribuir con las actuales medidas sanitarias de salud pública.

Agradecimientos: Al decano de la Facultad de Medicina Humana de la Universidad de San Martín de Porres, Dr. Frank Lizaraso Caparó, por el apoyo y licencias para el desarrollo del presente estudio.

Contribución de autoría: Echevarria Merino Hugo, Flores Asenjo Walter, Garay Ortega Juan y Salazar Granara Alberto concibieron la idea y el diseño del estudio. Echevarria Merino Hugo, Flores Asenjo Walter, Garay Ortega Juan, Roca Moscoso Max A llevaron a cabo la fase de reclutamiento y procesamiento de la información. Echevarria Merino Hugo, Flores Asenjo Walter, Garay Ortega Juan, Roca Moscoso Max A y Salazar Granara Alberto realizaron el análisis, la revisión crítica y la redacción de la versión final del artículo.

Fuentes de financiamiento: Este estudio ha sido financiado por los autores y por la Universidad de San Martín de Porres (USMP).

\section{REFERENCIAS BIBLIOGRÁFICAS}

1. Sandoval Paredes J, Sandoval Paz C. Uso de fármacos durante el embarazo. Horiz Med. 2018; 18(2): 71-9.

2. Kennedy DA, Lupattelli A, Koren G, Nordeng H. Herbal medicine use in pregnancy: results of a multinational study. BMC Complement Altern Med. 2013; 13: 355.

3. Dennehy C, Tsourounis C. Fitoterapia. En: Katzung, Masters, Trevor, eds. Farmacología Básica y Clínica. 12da ed. Leticia: Norma; 2013.

4. Organización Mundial de la Salud. Estrategia de la OMS sobre medicina tradicional 2014-2023 [Internet]. Ginebra: OMS; 2013. Disponible en: https://apps.who.int/iris/bitstream/ handle $/ 10665 / 9500 / 9789243506098$ spa.pdf; jsessionid=2D2F4EE4D 4E1B906BD801D3C877C902B?sequence $=1$

5. Tácuna-Calderón A, Moncada-Mapelli E, Lens-Sardón L, HuacchoRojas J, Gamarra-Castillo F, Salazar-Granara A. Estrategias de la Organización Mundial de la Salud en Medicina Tradicional y Reconocimiento de Sistemas de Medicina Tradicional. Rev Cuerpo Med HNAAA. 2020; 13(1): 101-2.

6. Ministerio de Salud. Ley de plantas medicinales peruanas [Internet]. Lima: MINSA; 1998. Disponible en: https://sisbib.unmsm.edu.pe/ bvrevistas/situa/1995_n6/ley_de_plant.htm

7. Palma-Gutiérrez E, Prado-Bravo C, Loja-Herrera B, Salazar-Granara A. Características fitoquímicas de muestras comerciales de maca en tres regiones de Perú. CIMEL. 2012; 17(2): 89-93.

8. Pawer-Pucurimay D, Park-Joon S, Roca-Moscoso M, Salazar-Granara A. Diferencias en la presencia de alcaloides y fenoles de cinco muestras de muña de expendio informal procedentes de mercados populares en Lima - Perú. Horiz Med. 2018; 18(3): 25-9.

9. Aguirre LG, Pereyra-Aguilar P, Silva-Arrieta-Ontaneda I, Alarcón-Urbina M, Palacios-Quintana M, Medina-Salazar H, et al. Consumo de plantas medicinales en usuarios del "Centro Integral del Adulto Mayor" de la Punta-Callao (Perú). Rev Fitoterapia. 2016; 16(2): 165-75.

10. Ernst $\mathrm{E}$. Herbal medicinal products during pregnancy: are they safe? BJOG. 2002; 109(3): 227-35.

11. da Silva Costa KC, Barbosa Bezerra S, Moreira Norte C, Nogueira Nunes LM, Moreira de Olinda T. Medicinal plants with teratogenic potential: current considerations. Braz J Pharm Sci. 2012; 48(3): 427-33.

12. Kshyamamayee Priyadarsini J, Aun Kumar M, Rajagopala S. Teratogenic effect of herbal drugs - A review. Int J Appl Ayurveda Res. 2018; 3(10): 1516-23.

13. Chávez-Alvarez RE, Arcaya Moncada MJ, García Arias G, Surca Rojas TC, Infante Contreras MV. Rescatando el autocuidado de la salud durante el embarazo, el parto y al recién nacido: representaciones sociales de mujeres de una comunidad nativa en Perú. Texto Contexto- Enferm. 2007; 16(4): 680-7.

14. Soares Cardoso B, Santana Amaral VC. The use of phytotherapy during pregnancy: a global overview. Cien Saude Colet. 2019; 24(4): 1439-50.

15. Pulido Acuña GP, Vásquez Sepúlveda PM, Villamizar Gómez L. Uso de hierbas medicinales en mujeres gestantes y en lactancia en un hospital universitario de Bogotá (Colombia). Index Enferm. 2014; 24(3): 1132-296.

16. Salazar-Granara A, Galvan M, Sanseverino MT, Abechea AM, Schuler-Faccini L. First inventory of herbal medicines consumed by Brazilian women during pregnancy. Reproductive Toxicology. 2013; 37(37): 77-87.

17. Nyeko R, Tumwesigye NM, Halage AA. Prevalence and factors 
associated with use of herbal medicines during pregnancy among women attending postnatal clinics in Gulu district, Northern Uganda. BMC Pregnancy Childbirth. 2016; 16(1): 296.

18. Illamola SM, Amaeze OU, Krepkova LV, Birnbaum AK, Karanam A, Job KM, et al. Use of Herbal Medicine by Pregnant Women: What Physicians Need to Know. Front Pharmacol. 2020; 10: 1483.

19. Orief YI, Farghaly NF, Abdelaziz Ibrahim MI. Use of herbal medicines among pregnant women attending family health centers in Alexandria. Middle East Fertil Soc J. 2014; 19(1): 42-50.

20. Ahmed M, Hwang JH, Choi S, Han D. Safety classification of herbal medicines used among pregnant women in Asian countries: a systematic review. BMC Complement Altern Med. 2017; 17: 489.

\section{Correspondencia:}

Alberto Salazar Granara

Dirección: Av. El Corregidor 1531, Las Viñas, La Molina. Lima, Perú.

Teléfono: 013652300 extensión 151

Correo electrónico: asalazarg@usmp.pe

\section{Recibido: 21 de setiembre de 2020}

Evaluado: 21 de diciembre de 2020

Aprobado: 26 de febrero de 2021

(c) La revista. Publicado por Universidad de San Martín de Porres, Perú. (c) By Licencia de Creative Commons Artículo en acceso abierto bajo términos de Licencia Creative Commons Atribución 4.0 Internacional. (http://creativecommons.org/licenses/by/4.0/)

\section{ORCID iDs}

Echevarria-Merino Hugo C https:// orcid.org/0000-0003-3605-3684

Flores-Asenjo Walter (1) https://orcid.org/0000-0002-5719-8009

Garay-Ortega Juan C https:// orcid.org/0000-0002-6174-4631

Roca-Moscoso Max A. C https://orcid.org/0000-0002-0805-6979

Salazar-Granara Alberto ittps: / / orcid.org/0000-0003-1996-3176 